\title{
Erinnern und Vergessen. Methodische Gedanken und mittelalterliche Perspektiven zu zwei Paradigmen am Schnittpunkt von Memoria und Geschichte
}

Erinnern und Vergessen - die komplementären Gegenstände dieses Bandes - gehören zu den grundlegenden sozialen Praktiken des Menschen. Historiker begegnen den beiden mit besonderem Interesse, nähern sich ihnen aus einer doppelten Perspektive: aus der methodischen Distanz objektivierender Wissenschaft und zugleich im Bewusstsein der subjektiven Nähe des erinnernden und vergessenden, am kollektiven Erinnerungsprozess beteiligten Individuums. In der Beschäftigung mit dem Erinnern und dem Vergessen reflektieren sie somit nicht allein wissenschaftliche Gegenstände, sondern ebenso die historisch-anthropogene Tiefe eigener sozialer Praxis. Erinnern und Vergessen führen Historiker ins Zentrum des Spannungsfeldes zwischen Distanz und Empathie gegenüber dem historischen Subjekt, zu dem sie in Dialog treten. Rhetorisch und erkenntnistheoretisch betrachtet deuten Erinnern und Vergessen zugleich auf das analogische Verhältnis des Historikers zu den Menschen vergangener Zeiten, deren Erfahrungen und Praktiken er versucht zu ergründen. Sie besitzen damit paradigmatisches Potential. ${ }^{1}$

Diesem Grundgedanken der paradigmatischen Dimension von Erinnern und Vergessen als Beispiele für auf Analogiedenken gestützte Zugangsformen zur Vergangenheit, in denen sich soziale Praxis und historisches Denken verbinden, möchte ich mich auf der Grundlage von Patrick Gearys Buch Phantoms of Remembrance und den Beiträgen zu diesem Band - keinesfalls summierend, eher eklektisierend - nähern, um an die bereits von Geary vorgenommene Problematisierung einer für unser Thema zentralen methodischen Trennlinie anzuknüpfen. Ich meine die von Maurice Halbwachs (1877-1945) etablierte Trennung zwischen Geschichte und (kollektivem) Gedächtnis.

Forschungen zu Erinnern und Vergessen als Motoren sozialer Prozesse, in deren Reihe Phantoms of Remembrance einen festen Platz einnimmt, haben einen wichtigen Beitrag zur Umdeutung des Spannungsfelds aus methodischer Distanz und Empathie zur produktiven Quelle des historischen Arbeitens geleistet. Patrick Geary verdeutlicht

1 Grundlegend ist die Definition des Paradigmas bei Aristoteles, allerdings als induktives Argument, vgl. Aristoteles, Rhetorik, II.2, 1375b25-35, Stuttgart 1999, S. 17. Die Definition des Paradigmas als analogische Erkenntnisform übernehme ich von Giorgio Agamben, Signatura rerum. Zur Methode, Frankfurt am Main 2009, S. 37.

Ә OpenAccess. (C) 2021 Gordon Blennemann, published by De Gruyter. (cc))BY-NC-ND This work is licensed under the Creative Commons Attribution-NonCommercial-NoDerivatives 4.0 International License. 
diese produktive Spannung in seinem Beitrag mit explizitem Blick auf die biographische Dimension seiner Arbeiten zur Erinnerungskultur, indem er die Beschäftigung mit einem Teil der eigenen Familiengeschichte des 19. Jahrhunderts als einen Ausgangspunkt dieser Forschungen herausstellt.

Dieser methodische Entwicklungsschritt war keineswegs selbstverständlich. Er ist vielmehr Teil einer breiter geführten, kritischen Auseinandersetzung mit methodischen Grundsätzen des Historismus, eine langfristige Debatte, deren Kontextualisierung und Entwicklung Walter Pohl in seinem Beitrag zu diesem Band nachzeichnet. Leopold von Ranke (1795-1886) forderte vom Historiker unbedingte Objektivität. Als Forscher wollte er sein „Selbst gleichsam auslöschen, und nur die Dinge reden, die mächtigen Kräfte erscheinen lassen“². Dieses Grundideal schloss neben der wissenschaftlichen Implikation des Historikers gegenüber seinem Forschungsgegenstand eine unausweichliche Restsubjektivität ein, durch die „im gegebenen Zusammenhang die Unterscheidung und Verknüpfung von Wert-Urteil und Erkenntnis-Urteil gelingen“3 konnte. Dieser Grundgedanke des Historismus war an strenge methodische Prämissen gebunden, die zwangsläufig zur Ablehnung von Formen außerwissenschaftlichen Schreibens über Geschichte führen musste, da diese als (zu) subjektiv empfunden wurden und damit anrüchig waren, eine Sichtweise, die eine weitere Trennlinie zog: jene zwischen Geschichte und Literatur ${ }^{4}$.

Maurice Halbwachs, dessen Gedanken zum Gedächtnis und seinen sozialen Bedingungen sowie zum Konzept des kollektiven Gedächtnisses einen zentralen Ausgangspunkt für die wissenschaftliche Beschäftigung mit Erinnern (und Vergessen) als gesellschaftliche Phänomene darstellen, wehrte sich gegen die Grundannahmen des Historismus, indem er der systematisierenden und reflektierenden, dadurch aber auch manipulativen Historie die Natürlichkeit und Offenheit des kollektiven Gedächtnisses gegenüber stellte. Halbwachs beschrieb das Verhältnis der beiden als Dichotomie, als schnittmengenlose Gegenüberstellung von geradezu feindlicher Natur, da er in der Historie ein bürgerliches Elitenphänomen sah, dessen Träger den demokratischen Wert und die ideologische Ungebundenheit des kollektiven Gedächtnisses bewusst verfälschten. Auch wenn dieser gesellschaftskritische Ansatz im Zeitkontext der 20er und 30er Jahre des 20. Jahrhunderts verständlich erscheint, so hat doch die Idee einer eindeutigen Abgrenzung von kollektivem Gedächtnis und Geschichte den nachfol-

2 Leopold von Ranke, Sämtliche Werke, Bd. 15: Englische Geschichte vornehmlich im siebzehnten Jahrhundert. Zweiter Band, Leipzig 1870, S. 103.

3 So Otto Gerhard Oexle, Memoria als Kultur, in: Memoria als Kultur, hrsg. von dems., Göttingen 1995 (Veröffentlichungen des Max-Planck-Instituts für Geschichte, 121), S. 9-78, das Zitat S. 11-12, dazu umfassend auch ders., Von Nietzsche zu Max Weber: Wertproblem und Objektivitätsforderung der Wissenschaft im Zeichen des Historismus, in: Rechtsgeschichte und theoretische Dimension. Forschungsbeiträge eines Rechtshistorischen Seminars in Stockholm im November 1986, hrsg. von Claes Peterson, Stockholm 1990, S. 111-145.

4 Dazu die programmatische Monographie Ivan Jablonka, L'histoire est une littérature contemporaine. Manifeste pour les sciences sociales, Paris 2014 (Librairie du Xxie siècle). 
genden Forschergenerationen eine definitorische Hypothek auferlegt, die in der Tat, wie Walter Pohl betont, paradox erscheint, wenn man davon ausgeht, dass Historiker - wie eingangs angedeutet - vermittelnd zwischen beiden stehen. Ihre Entwicklung, Gestaltung und wechselseitige Wirkung wären somit als gesamtgesellschaftlicher Prozess $\mathrm{zu}$ deuten und dies nicht etwa erst in modernen demokratischen Zusammenhängen.

Denn Historiker in Zeiten vor der sogenannten Verwissenschaftlichung der Geschichtsschreibung taten sich um vieles leichter mit solchen Fragen. Und es ist im Grunde verwunderlich, dass die etwa 350-jährige Methodentradition seit Beginn der Aufklärung noch immer mehr wiegt als die historiographische Praxis der rund 2200 Jahre zuvor. Lesen wir etwa beispielhaft den Prolog einer der zentralen biographisch-historiographischen Erinnerungstexte des Frühmittelalters - Einhards Vita Karoli Magni - so begegnen wir dort einem Autor der sein Wirken als persönlichen, durchaus subjektiven Erinnerungsakt in einen gesamtgesellschaftlichen Bedürfnisrahmen einordnet, die Bedeutung seines Berichts als Augenzeuge herausstellt, zugleich die Wertigkeit paralleler Darstellungen akzeptiert, nicht zuletzt aber auch seinen Text - bei aller Bescheidenheitstopik - selbstbewusst als an großen Vorbildern orientierte, rhetorisch-sprachliche Leistung präsentiert ${ }^{5}$.

Im Hinblick auf das Erinnerungsbild Karls weist Einhard sich selbst eine Mittlerfunktion zwischen den verschiedenen Traditionssträngen und den an der Erinnerung beteiligten Akteuren zu, welche die vom Autor ausgehende rhetorisch-persuasive Dimension im Sinne des prodesse et delectare keineswegs verschleiert. Gerade deshalb sollte man den im Prolog durchscheinenden Anspruch auf eine breitere gesellschaftliche, ja öffentliche Wirkung ernst nehmen, denn Rhetorik war und ist - zumindest auf die Adressaten bezogen - kein Elitenphänomen.

Damit stellt die Tatsache, dass Einhard als Autor individuell oder als Teil einer Interessengemeinschaft durch die Karlsbiographie gewiss zeitgebunden politisch handelte, die Verbindungen seines Textes zum sozialen Gedächtnis seiner Zeit nicht in Frage. Ebenso stehen die Kontextgebundenheit und Intentionalität der Biographie nicht im Widerspruch zu ihrem (narrativen) Anteil an der Gestaltung von Vergangenheit als soziale Konstruktion im Hinblick auf ein frühmittelalterliches Gesellschaftsgefüge, das sich nicht in Gruppendynamiken und Teilöffentlichkeiten erschöpft ${ }^{6}$.

5 Einhard, Vita Karoli Magni, hrsg. von Oswald Holder-Egger, in: MGH SS rer. Germ. in usum schol. [25], Hannover, Leipzig 1911, S. 1-2, vgl. dazu Steffen Patzold, Einhards erste Leser. Zu Kontext und Darstellungsabsicht der „Vita Karoli“, in: Viator Multilingual, 42, 2011, S. 33-55.

6 Zur Problematisierung des Öffentlichkeitsbegriffs im Hinblick auf das Frühmittelalter zusammenfassend Hedwig Röckelein, Reliquientranslationen nach Sachsen im 9. Jahrhundert. Über Kommunikation, Mobilität und Öffentlichkeit im Frühmittelalter, Stuttgart 2002 (Beihefte der Francia, 48), S. $43-47$. 
Das Beispiel Einhards zeigt, wie vorwissenschaftliche Sichtweisen auf das Verhältnis von kollektivem oder sozialem Gedächtnis und Geschichtsschreibung, methodische Grundannahmen der modernen Geschichtswissenschaft (zumindest aus meiner Sicht) relativieren können, es sei denn, man sieht in der Methodendiskussion des 18. und vor allem 19. Jahrhunderts eine unhintergehbare Trennlinie zu vormodernen Traditionen. Was bei Einhard - noch ist man geneigt zu ergänzen - als Komplementarität erscheint, wird bei Halbwachs zur (auch gesellschaftlich begründeten) Dichotomie, wobei die Trennung von kollektivem Gedächtnis und Historie - wie bereits betont vor allem auch aus der kritischen Auseinandersetzung mit der zeitgenössischen Geschichtswissenschaft hervorging. Dabei muss Halbwachs eine gewisse terminologische Inkohärenz eingestehen: In seinem unvollendet geblieben Buch La mémoire collective oszilliert er auffällig zwischen der Bezeichnung histoire und mémoire historique und räumt ein, dass die Bezeichnung „historisches Gedächtnis nicht sehr glücklich gewählt sei, da sie zwei Begriffe“ zusammenführe, „die sich in mehr als einem Punkt“" widersprächen”.

Der gewaltsame Tod im Konzentrationslager Buchenwald 1945 nahm Halbwachs die Möglichkeit, diesen Punkt kritisch zu vertiefen. Bei aller Bewunderung und Inspiration hat die Forschung zu Gedächtnisformen und Gedächtniskulturen nach 1945 unter anderem aber an dieser rhetorischen und logischen Bruchstelle im Werk Halbwachs' angesetzt - mit prominenten Ausnahmen, wie Pierre Nora, der die Idee der Dichotomie zwischen kollektivem Gedächtnis und Geschichte politisierte und weiter vertiefte ${ }^{8}$.

Jan Assmanns behutsam kritische Beschäftigung mit Halbwachs aus der Sicht der Altertumswissenschaften bestätigt zwar zunächst aus dessen Logik heraus den Gegensatz von Gedächtnis und Historie, trennt sich aber von dem damit verbundenen Gegensatz zwischen Gedächtnis und Tradition und betont die fließenden Übergänge zwischen diesen beiden Polen, die im Ergebnis ebenso zwischen Gedächtnis und „Geschichtsschreibung als Symptom veränderter Erinnerungskultur“ bestehen 9 . Kommunikatives und kulturelles Gedächtnis - die beiden Schlüsselkategorien im Werk Aleida und Jan Assmanns - geben diesen fließenden Übergängen einen konzeptuellen Rahmen, da beide Gedächtnisformen als aufeinander bezogene Entwicklungsschritte bestimmt werden ${ }^{10}$.

7 Maurice Halbwachs, La mémoire collective, Paris 1950, S. 45 (meine Übersetzung): De tout ce qui précède il résulte bien que la mémoire collective ne se confond pas avec l'histoire, et que l'expression : mé moire historique, n'est pas très heureusement choisie, puisqu'elle associe deux termes qui s'opposent sur plus d'un point.

8 Pierre Nora, Mémoire collective, in: La Nouvelle Histoire, hrsg. von Roger Chartier, Jacques Le Goff und Jacques Revel, Paris 1978, S. 398-401.

9 Jan Assmann, Das kulturelle Gedächtnis. Schrift, Erinnerung und politische Identität in frühen Hochkulturen, München 1992, das Zitat S. 299 sowie zur Auseinandersetzung mit Halbwachs’ Gegenüberstellungen von Gedächtnis und Geschichte und Gedächtnis und Tradition S. 42-45.

10 Neben der vorgenannten Monographie Jan Assmanns siehe beispielhaft Aleida Assmann, Erinnerungsräume. Formen und Wandlungen des kulturellen Gedächtnisses, München 1999. 
Patrick Geary hat in Phantoms of Remembrance - ebenfalls in Auseinandersetzung mit Maurice Halbwachs - sein Augenmerk auf eben diese fließenden Übergänge und die in ihnen wirkenden Formen der Vermittlung zwischen Gedächtnis und Geschichte gelegt. Wie bei Jan Assmann rücken so zwangsläufig die Prozesshaftigkeit und damit Fragen der Materialität und Medialität von Erinnern in den Mittelpunkt.

Das für diesen Band zentrale Vergessen ordnet Geary in den breiteren Rahmen solcher sozialen Erinnerungsprozesse ein. Mit Blick auf die Zeitgeschichte hat auch Aleida Assmann jüngst die politisch-gesellschaftliche Bedeutung des Vergessens als aktiver Prozess der Fokussierung und Selektion unterstrichen ${ }^{11}$. Generell hat sich die historische, nicht zuletzt die mediävistische Forschung dem Vergessen als Teilaspekt von Erinnerungstechniken und Erinnerungskulturen gewidmet ${ }^{12}$. Als eigenständiger Fokus der historisch-kulturwissenschaftlichen Forschung hat sich das Vergessen allerdings erst in den letzten Jahren etabliert. Die Arbeiten von Sebastian Scholz sowie vor allem von Gerald Schwedler ${ }^{13}$, deren Grundlinien in der Einleitung zu diesem Band dargelegt werden, haben wesentlich zu dieser thematischen Rückung beigetragen. Sie geben der Deutung des Vergessens als politisch-sozialer Prozess nicht allein mediävistisch begründete historische Tiefe. Vor allem setzen sie mit den beiden Begriffen der selektiven Erinnerung und der kreativen Selektion methodisch-konzeptuelle Bezugspunkte, deren analytische Wirkmächtigkeit in den Beiträgen zu diesem Band vor Augen geführt wird. Ich möchte die Ergebnisse dieser Beiträge auf einige Aspekte hin zuspitzen.

Die Herausgeber des Bandes identifizieren in ihrer Einleitung die Untersuchung von Wissenskonfigurationen und ihrer sozialen Kontexte als zentralen thematischen Schwerpunkt der Beschäftigung mit Selektionsprozessen als Teil frühmittelalterlicher Erinnerungskultur. Durch die Fokussierung auf Texte als Medien solcher Selektionsprozesse ergeben sich daraus nicht zuletzt auch Perspektiven für eine kultur- und vor allem sozialgeschichtliche Neuorientierung traditioneller textgeschichtlicher Forschung.

Die Beiträge bilden eine große Bandbreite von Texttypen als Medien kreativer Selektion ab, die von Historiographie und Hagiographie, über Personenlisten, Genealogien, Briefe, Traktate und kirchliche und weltliche Rechtstexte zu monastischen Normentexten reicht. Solche modernen Gattungszuweisungen kennen allerdings gerade im Hinblick auf unsere Thematik Grenzen. Denn das Potential der Texte für die Gestaltung von Vergangenheitsbildern war wesentlich an ein offenes Textverständnis

11 Aleida Assmann, Formen des Vergessens, Göttingen 2016 (Historische Geisteswissenschaften. Frankfurter Vorträge, 9).

12 Vgl. dazu den Forschungsüberblick in Patrick Gearys Beitrag zu diesem Band S. 17-20.

13 Gerald Schwedler, Vergessen, Verändern, Verschweigen und Damnatio memoriae im frühen Mittelalter, Köln u. a. 2021 (Zürcher Beiträge zur Geschichtswissenschaft 9). 
gebunden, das nicht nur für das Mittelalter spezifisch ist ${ }^{14}$. Die von Patrick Geary untersuchten Chartulare, die um historiographische und hagiographische Elemente ergänzt wurden, sind hierfür ein klassisches Beispiel. Ganz ähnlich verhält es sich mit memorierenden Personenlisten als Teil historiographischer Narration (Philippe Dépreux). Genealogien konnten um narrative Elemente ergänzt und damit zu einer Form von Geschichtserzählung umgeformt werden (Helmut Reimitz). Die serielle Logik von Sammelhandschriften macht sich dasselbe offene Textverständnis zu eigen, indem etwa Rechtstexte ganz unterschiedlicher Natur zu einem kohärenten Rechtsinstrument oder Rechtsmonument zusammengebunden wurden (Michael Eber - Stefan Esders - David Ganz - Till Stüber). Im Falle von Namenslisten und Genealogien hatte die serielle Form einen wesentlichen Einfluss auf die argumentative Kraft des Vergangenheitsbilds, das durch die Personenabfolge getragen wurde. Brüche in der Abfolge konnten leicht kaschiert werden, um Kontinuität zu suggerieren. Die Form des Texts wird hier nahezu zum Argument sui generis. Dies hing nicht zuletzt auch damit zusammen, dass solche Listen auf historische, in erster Linie biblische Modelle wie Christusgenealogien und Patriarchenlisten als autoritative Vorlagen rekurrierten.

Die zum Teil breite handschriftliche Streuung der untersuchten Texte und Textkomplexe macht deutlich, dass solche Strategien im Zusammenhang ausgreifender Textnetzwerke entwickelt und verhandelt wurden. Damit stellt sich die Frage nach der Wirkung und dem intendierten oder tatsächlichen Publikum. Die zusammengetragenen Fallbeispiele illustrieren komplexe Formen der Vergangenheitskonstruktionen, die ausnahmslos an schriftliche Fixierung gebunden waren, was nicht zuletzt ihre Verbreitung sicherte. Inhaltliche Veränderungen und Tilgungen, die etwa in den fränkischen Königsgenealogien Aktualisierungen des Geschichtsbildes sichtbar machen, werfen allerdings auch die Frage auf, inwiefern solche Veränderungen vor der Verschriftlichung diskutiert wurden. Für monastische Gemeinschaften sind solche mündlichen Aushandlungsprozesse im Hinblick etwa auf normative Fragen (Jörg Sonntag) aber auch für hagiographische und historiographische Texte dokumentiert, die in der Gemeinschaft zur Diskussion gestellt wurden ${ }^{15}$. Dies gilt ebenso für politische Entscheidungsprozesse im Rahmen von Versammlungen ${ }^{16}$.

Hier zeichnen sich Formen partizipativer Literalität $t^{17} \mathrm{ab}$, welche die zentrale Bedeutung von Schriftlichkeit, aber auch ihr komplementäres, keinesfalls hierarchi-

14 Dazu grundlegend Roland Barthes, De l'œuvre au texte, in: ders., Euvres complètes. hrsg. von Éric Marty, Bd. 3: Livres, textes, entretiens 1968-1971, Paris 2002, S. 908 -916.

15 Vgl. das Beispiel bei Patrick Geary, Phantoms of Remembrance. Memory and Oblivion at the End of the First Millennium, Princeton (NJ) 1994, S. 11-12.

16 Dazu allgemein den Band Streit am Hof im frühen Mittelalter, hrsg. von Matthias Becher und Alheydis Plassmann, Göttingen 2011 (Super alta perennis, 11).

17 Zum Begriff de Literalität in frühmittelalterlichen Zusammenhängen The uses of literacy in early medieval Europe, hrsg. von Rosamond McKitterick, Cambridge u.a. 1990 sowie zum weiteren thematischen Rahmen mit reicher Bibliographie New approaches to medieval communication, hrsg. von Marco Mostert, Turnhout 1999 (Utrecht Studies in medieval literacy 1). 
sches Verhältnis zu Mündlichkeit in der frühmittelalterlichen Welt unterstreichen. Und wir sollten nicht vergessen, dass der mündliche performative Vortrag eines literarischen Textes durch einen geschulten Lektor (auch jenseits des Frühmittelalters) hoch geschätzt war, weshalb Paul Zumthor (1915-1995) den Begriff der Vokalität dem Begriff der Mündlichkeit vorzog ${ }^{18}$. Dieselbe Wertigkeit von Vokalität zeigt sich in Formen ritualisierter, näherhin liturgischer Memoria. Es sei etwa daran erinnert, dass für Augustinus Verstorbene durch die mündliche Äußerung ihres Namens in liturgischen Kontexten gegenwärtig wurden ${ }^{19}$. Die Rasur eines Namens in einem als irdische Analogie zum himmlischen Lebensbuch gedachten Liber memorialis kam in diesem Sinne der physischen Tilgung aus der Gemeinschaft der Lebenden und der Toten gleich, die ekklesiologisch betrachtet dem Ausschluss aus der Gemeinschaft der Heiligen entsprach ${ }^{20}$.

Angesichts der angedeuteten Perspektiven kollektiver Aushandlungsprozesse wäre letztlich auch zu fragen, inwiefern textlich greifbare Vergangenheitsbilder in einem öffentlichen Raum diskutiert und konstituiert wurden. Wenn die Pippiniden nach der Thronusurpation von 751 durch Aneignung vorhergehender Traditionen fränkischer Königsgenealogien ihre eigene familiäre Memoria als Teil aristokratischer Erinnerungskultur verköniglichten, dann wird darin vielleicht sogar beispielhaft greifbar, wie sich um den Aspekt der selektierender und transformierender Erinnerung ein differenziertes Verständnis von privatem und öffentlichem Raum formte, zwei Kategorien, die in die Untersuchung antiker Gesellschaften problemlos integriert werden $^{21}$.

Dies führt uns zurück zu den bereits für Einhard und seine Karlsvita thematisierten gesellschaftlichen Bezugsebenen, die das Handeln jener Autoren bestimmten, die aktiv - ob als Individuum oder Gruppe - Vergangenheitsbilder im Rahmen kreativer Selektionsprozesse gestalteten. Die gestalterische Kraft von Individuen, die im Sinne der Entwicklung eines kulturellen Gedächtnisses mitunter weit über ihren Zeitkontext hinausreicht, ist zumeist nur für die namentlich Bekannten näher zu fassen. Isidor von Sevilla wird als politisch motivierter Spiritus rector der Damnatio memoriae des Westgotenkönigs Gundemar greifbar (Michael J. Kelly). Gregor von Tours bestimmt bis heute durch ein Werk unser Bild des 6. Jahrhunderts, das ein heilsgeschichtlich fundiertes, aber auch aus der Lebenserfahrung des Autors ge-

18 Paul Zumthor, La lettre et la voix. De la littérature médiévale, Paris 1987.

19 Augustinus, De cura pro mortuis gerenda, 6, hrsg. von Joseph Zycha, in: Corpus Scriptorum Ecclesiasticorum Latinorum 41, Prag u. a. 1900, S. 630-631.

20 Vgl. Sebastian Scholz, ,Durch eure Fürbitten ist er Gefährte der Heiligen'. Grabinschriften als Ausdruck des Totengedenkens im Mittelalter, in: Bücher des Lebens - lebendige Bücher. Katalog, hrsg. von Peter Erhart und Jakob Kuratli, St. Gallen 2010, S. 70 - 82.

21 Public and Private in Ancient Mediterranean Law and Religion, hrsg. von Ando Clifford und Jörg Rüpke, Berlin, München, Boston 2015 (Religionsgeschichtliche Versuche und Vorarbeiten 65). 
speistes sozioekklesiologisches Modell für seine Zeit entwarf ${ }^{22}$. Die Selektionsentscheidungen Gregors (und Fredegars) gegenüber den ihm verfügbaren Überlieferungen werden dabei als eine die Geschichtsschreibung geradezu definierende Grundbedingung sichtbar (Gerald Schwedler), ein Nachhall Herodot'scher Arbeitsweisen. Für Jonas' von Bobbio Wirken als Autor hat die neueste textgeschichtliche Forschung für die vorkarolingische Zeit recht einzigartige Perspektiven einer biographischen Verortung eröffnet, die vor allem zeigen, wie sehr seine Darstellungen der monastischpolitischen Entwicklungen um die Figur Columbans zeitbezogene Konstruktionen und Beiträge zu aktuellen Debatten um theologische Fragen waren (Ian Wood), die in der hagiographischen Narration offensichtlich oder latent greifbar werden.

Gerade das Beispiel Jonas‘ von Bobbio, der - vermittelt durch die katholische Geschichtsforschung des 19. Jahrhunderts - zum Kronzeugen des 7. Jahrhunderts wurde, zeigt, wie sehr wir Historiker selbst durch unsere Selektionsentscheidungen eine im Prinzip reiche Überlieferung dem Vergessen Preis geben. Das an hagiographischen Schriften nicht eben arme 7. Jahrhundert ist dadurch ein Stück weit zum Jahrhundert der Hagiographie Jonas‘ von Bobbio geworden. Anders gesagt: Hinter solchen namentlich bekannten Autoren, die bereits im Mittelalter gelöst von ihren Biographien zu Autoritäten wurden, steht eine anonyme Masse von Männern und Frauen, die mit gleichem Talent Anteil an der Erinnerungskultur ihrer Zeit nahmen und dies nicht allein in Form von historiographischen und hagiographischen Narrativen. Rechtssammlungen etwa zeigen wie durch chronologische oder thematische Ordnungsprinzipien die historische Verortung von Rechtstraditionen betont oder verdeckt werden konnte, um durch die Verbindung der verschiedenen Überlieferungen im frühmittelalterlichen Kontext an die spätantike Rechtsvergangenheit der (zumindest partiellen) Einheit von imperialem und kirchlichen Recht anzuknüpfen. Experten der Ordnung und schriftlichen Fixierung konnte somit bewusst die Rolle zugewiesen werden, die unterschiedlichsten ideologischen Traditionsstränge zu einer im Zeitkontext relevanten Narration zu synthetisieren. Wenn wir davon ausgehen, dass solche narrativen Konstruktionen Teil einer öffentlichen Diskussion um Erinnerungsbilder und Repräsentationen von Vergangenheit waren, dann ist eine solche Rollenzuweisung nicht verwunderlich, denn Experten kommt häufig die Aufgabe zu, öffentliche Diskurse zu katalysieren. Es ist ebenso wenig verwunderlich, dass eine solche Aufgabe mitunter Outsidern zugeschrieben wurde, wenn wir etwa an das Wirken des Italieners Venantius Fortunatus als Hagiograph in der Gallia denken.

Ein Desiderat für künftige Forschungen wäre noch $\mathrm{zu}$ formulieren: Historiker beschäftigen sich noch immer zu wenig mit der Bedeutung materieller und bildlicher Zeugnisse als Erinnerungsmedien und Mittel zur Vergangenheitskonstruktion. Im Band kommt der Anteil der Münzen am hochmittelalterlichen Erinnerungsbild Karls des Großen zur Sprache, die zudem auf die Bedeutung der Wechselwirkung ver-

22 Dazu grundlegend Martin Heinzelmann, Bischof Gregor von Tours (538-594). „Zehn Bücher Geschichte“: Historiographie und Gesellschaftskonzept im 6. Jahrhundert, Darmstadt 1993. 
schiedener Medien verweisen (Manfred Groten). Ein klassisches Beispiel aus spätantik-frühmittelalterlichen Zusammenhängen wären die ostgotischen Prozessionsmosaiken in S. Apollinare Nuovo in Ravenna. Es ist wahrscheinlich, dass die ursprüngliche Ausstattung Hofmitglieder Theoderichs des Großen darstellte und aus diesem Grund in justinianischer Zeit politisch motiviert durch Heiligenprozessionen ersetzt wurde. Dabei blieben allerdings einzelne Teile - vor allem Hände - der ursprünglichen Figuren sozusagen als Sichtbarmachung der Damnatio memoriae bestehen ${ }^{23}$.

Die Einbindung von Bildzeugnissen bedeutet mehr als eine bloße Bereicherung des Quellenmateriales. Sie eröffnet vor allem Zugänge zu Methodentraditionen der Bild- und Kunstwissenschaft, die für unsere Thematik zentral sind. Ich denke hier vor allem an die bildbezogenen kulturwissenschaftlichen Ansätze Aby Warburgs (18661929), der als erster das Konzept des sozialen Gedächtnisses einführte ${ }^{24}$. Warburg interessierte sich vor allem für die bildliche Tradition universaler Gesten und Mimiken des Menschen, Pathosformeln, in denen als Teil der Geschichte Grundformen der körperlichen Äußerung des Menschen als Nachleben - so der zweite von Warburg formulierte Schlüsselbegriff in diesem Zusammenhang - greifbar werden ${ }^{25}$. Bilder sind damit zentrale Träger des sozialen Gedächtnisses, in denen vor allem auch unbewusste, latente Erinnerungsmomente greifbar werden - ein Gedanke der durchaus Parallelen zur von Patrick Geary gebrauchten Metapher der ,phantoms of remembrance' aufweist ${ }^{26}$.

Warburgs Ansatz der Bilddeutung ist nicht allein ästhetische Theorie, sondern besitzt eine dezidiert politische und soziale Dimension. Denn der Begriff der Pathosformel definiert universale menschliche Gesten als Erinnerungsträger individueller wie kollektiver menschlicher Erfahrungen. Als Teil des kulturellen Gedächtnisses geht somit von Pathosformeln eine dauerhafte Wirkung aus. Georges Didi-Huberman hat diesen Gedanken kürzlich in einer umfangreichen Ausstellung auf die Geste der Erhebung (soulèvement) zugespitzt ${ }^{27}$. Warburg setzte somit den Rahmen für eine historische Bildforschung, in der Gedächtniskonzepte eine zentrale Rolle spielen.

Der von Warburg unmittelbar inspirierte Percy Ernst Schramm (1894-1970) hat dessen Ansätze für die mediävistische Geschichtswissenschaft verfügbar gemacht, sie

23 Dazu zusammenfassend Carola Jäggi, Ravenna. Kunst und Kultur einer spätantiken Residenzstadt. Die Bauten und Mosaiken des 5. und 6. Jahrhunderts, Regensburg 2016, S. 180-182.

24 Aby Warburg, Schlangenritual. Ein Reiseberich, Berlin 1988.

25 Anfänglich Aby Warburg, Dürer und die italienische Antike (1905), in: ders., Werke in einem Band, hrsg. von Perdita Ladwig, Martin Treml und Sigrid Weigel, Frankfurt am Main 2010, S. 176-183, realisiert vor allem im Bildatlas Mnemosyne, dazu ders., Mnemosyne. Einleitung (1929), in: ebd., S. 629639 und ders., Mnemosyne I., in: ebd., S. 640 - 646. Zur Wissensarchäologie der Waburg'schen Ideen Georges Didi-Huberman, L’image survivante. Histoire de l'art et temps des fantômes selon Aby Warburg, Paris 2002.

26 Zum Konzept der Latenz auch Latenz. Blinde Passagiere in den Geisteswissenschaften, hrsg. von Hans Ulrich Gumbrecht und Florian Klinger, Göttingen, Oakville (CT) 2011.

27 Soulèvements, hrsg. von Georges Didi-Huberman, Paris 2016. 
zugleich aber auf den Aspekt der politischen Ikonologie eingeengt ${ }^{28}$. Die breite soziokulturelle Ausrichtung Warburgs, vor allem sein Interesse an der Verflechtung von Bildtradition und sozialem Gedächtnis, die nicht zuletzt in Ernst Cassirers (18741945) Philosophie der symbolischen Formen ein systematisierend analytisches Pendant fand ${ }^{29}$, ist dadurch - zumindest für die mittelalterliche Geschichte - nachhaltig verschleiert worden. Dabei konnte für andere Bereiche der historischen Kulturwissenschaften im Rückgriff auf Warburg gezeigt werden, wie sehr Bilder und Texte und die mit ihnen verbundenen medialen Strategien komplementär oder konkurrierend gesellschaftliche Erinnerungsprozesse bestimmen ${ }^{30}$. Wenn wir den Beitrag der Historie zur Konstruktion des sozialen Gedächtnisses - wie in diesem Band vorgeschlagen - vor allem in der Wirkung medialer Selektionsprozesse fassen, dann stellten Bilder hierfür ganz eigene Strategien zur Verfügung. Die vergleichende Betrachtung von Bild- und Textstrategien im Rahmen solcher Selektionsprozesse dürfte somit - im Sinne Warburgs - weitergehende Perspektiven für das Verständnis frühmittelalterlicher Erinnerungskulturen und deren soziale Kontexte eröffnen.

Beunruhigt - wie Maurice Halbwachs - von den aufwallenden Totalitarismen seiner Zeit formulierte Walter Benjamin (1892-1940), „Vergangenes historisch artikulieren“ heiße nicht, „es erkennen ,wie es denn eigentlich gewesen ist“.” Es heiße, „sich einer Erinnerung bemächtigen, wie sie im Augenblick einer Gefahr aufblitzt. “31 In deutlicher Abgrenzung vom Historismus entwirft Benjamin hier um den Begriff der Erinnerung eine geschichtswissenschaftliche, vor allem aber auch gesellschaftliche Handlungsmaxime, welche die bei Aby Warburg auf das Phänomen der Pathosformel zentrierte Korrelation von sozialem Gedächtnis, Geschichte und Gegenwart fortdenkt. Ich möchte behaupten, dass der eingangs formulierte Vorschlag, Erinnern und Vergessen als auf Analogiedenken basierende konzeptuelle Zugänge zur Vergangenheit zu denken, Perspektiven einer solchen Bemächtigung von Erinnerung eröffnet. Analogie wäre dabei - entgegen der üblichen sinnreduzierenden Praxis - nicht als schlichte Wesensgleichsetzung zu verstehen, durch die sich historisches Denken in Anachronismen verfänge. Sie wäre vielmehr auf der Grundlage rhetorischer und erkenntnistheoretischer Traditionen als Relationsgleichheit zweier Objekte zu definieren, die

$28 \mathrm{Zu}$ den persönlichen und gedanklichen Beziehungen zwischen Aby Warburg und Percy Ernst Schramm siehe Lucas Burkart, Verworfene Inspiration. Die Bildgeschichte Percy Ernst Schramms und die Kulturwissenschaft Aby Warburgs, in: Bilder als historische Quellen? Dimension der Debatten um historische Bildforschung, hrsg. von Jens Jäger und Martin Knauer, München 2009, S. 71-96.

29 Ernst Cassirer, Philosophie der symbolischen Formen, 3 Bde, Hamburg 2010. Zum komplexen Verhältnis zwischen Warburg und Cassirer Didi-Huberman, L'image survivante (wie Anm. 25), Paris 2002, S. $433-451$.

30 Siehe beispielhaft den Band Märtyrer-Porträts. Von Opfertod, Blutzeugen und heiligen Kriegern, hrsg. von Sigrid Weigel, München u.a. 2007.

31 Walter Benjamin, Über den Begriff der Geschichte, hrsg. von Gérard Raulet, Frankfurt am Main 2010 (Kritische Gesamtausgabe 19), S. 75. 
nicht auf den Eigenschaften der Objekte selbst, sondern auf den wechselseitigen Beziehungen zwischen diesen Eigenschaften beruht ${ }^{32}$. Aby Warburgs Konzept der Pathosformel wäre ein Ansatzpunkt, um darüber nachzudenken, wie solch ein komplexes Analogieverständnis für historisches Denken zu nutzen wäre. Es käme darauf an, im Sinne Foucault'scher Wissensarchäologie die Beziehungen zwischen Analogiedenken und Praktiken des Erinnerns und Vergessens zu historisieren. Die Autoren dieses Band bieten hierfür aus der Perspektive des Früh- und Hochmittelalters sinnvolle Anknüpfungspunkte.

32 So die Definition bei Hans Höffding, Der Begriff der Analogie, Leipzig 1924, S. 7. 
